\title{
Which comes first: the antigen or the adjuvant?
}

\author{
Sallie R. Permar ${ }^{1}$ and Herman F. Staats ${ }^{2}$
}

\author{
1Department of Pediatrics and 2Department of Pathology, Human Vaccine Institute, Duke University Medical Center, Durham, North Carolina, USA.
}

\begin{abstract}
Recent attempts to develop an HIV-1 vaccine indicate that viral replication can be limited by the induction of viral-specific $T$ cell responses; however, recent trials of vaccine candidates designed to target $\mathrm{CD8}^{+} \mathrm{T}$ cell responses were unsuccessful. In this issue, Sui and colleagues used a nonhuman primate model to investigate the effect of various vaccine adjuvants on the efficacy of SIV immunization. Unexpectedly, Sui et al. discovered that animals given adjuvant alone in the absence of SIV antigen exhibited a pronounced decrease in viral load following viral challenge. Vaccination with viral antigens combined with adjuvant correlated with the expansion of a population of cells with similarity to myeloid-derived suppressor cells (MDSCs) that may have suppressed vaccine-elicited $T$ cell responses. Together, these results suggest that both innate and adaptive vaccine-elicited immune responses will need to be considered in future HIV-1 vaccine development.
\end{abstract}

The HIV-1 vaccine field has debated the importance of eliciting strong functional antibody responses to prevent viral invasion of target cells versus eliciting potent $\mathrm{T}$ cell responses to kill virus-infected cells. This debate has converged on the consensus that both arms of the immune response will likely be necessary to achieve effective HIV-1 vaccination $(1,2)$. Additionally, the field has turned toward investigating TLR agonists and other adjuvants to enhance dendritic cell antigen presentation and augment vaccine-elicited responses (3-8). Despite repeated attempts to produce an efficacious HIV-1 vaccine, our knowledge of the adjuvant-specific impact on both the humoral and cellular arms of vaccine-elicited immune responses is still nascent. Moreover, the necessity of HIV-1 vaccine candidates to engage the innate immune system is an ongoing area of investigation $(9,10)$.

\section{Vaccine adjuvant: \\ an unexpected effect}

In this issue, Sui et al. have demonstrated an unexpected effect of vaccine adjuvants on the development of T cell-mediated immunity that is potentially explained by innate immune regulation of vaccine-elicited cellular responses (11). While the investigated application of an adjuvanted peptide-prime/modified vaccinia Ankara (MVA) boost simian immunodeficiency virus (SIV)

Conflict of interest: The authors have declared that no conflict of interest exists.

Citation for this article: J Clin Invest. doi:10.1172/ JCI76263. vaccine approach did not protect against SIV acquisition in the nonhuman primate rectal challenge model, there was a notable decrease in viral load at set point in animals that received the SIV vaccine in combination with an adjuvant that modulates the $\mathrm{T}$ cell coinhibitory molecule PD1. Surprisingly, animals that received adjuvant alone exhibited a more pronounced decrease in viral load at both peak and set-point viremia compared with that observed in animals receiving adjuvant in combination with vaccine antigen. This adjuvant-protective effect was especially pronounced in animals that expressed the protective $\mathrm{MHC}$ class I allele Mamu-A*01. Moreover, this observed virus control was abrogated by depletion of $\mathrm{CD}^{+} \mathrm{T}$ cells. Thus, Sui and colleagues hypothesized that innate regulatory mechanisms limit the MHC class Irestricted cytotoxic $\mathrm{T}$ cell responses elicited by the vaccine antigens delivered with adjuvant compared with responses seen with adjuvant alone. In fact, Sui et al. demonstrated a correlation between the set-point viral load and the peripheral expansion of a myeloid cell population that phenotypically resembled myeloid-derived suppressor cells (MDSCs), suggesting an innate dampening of the vaccine-elicited responses following viral challenge (11). This finding raises two important points to consider in future vaccine strategies for HIV-1 and other pathogens: (a) what is the impact of novel vaccine adjuvants on global immunity following vaccination, and (b) should vaccine strategies avoid stimulation of innate immune cell populations such as MDSCs that could dampen vaccine-elicited immune responses?
The results from the study by Sui and colleagues (11) extend previous observations that TLR ligands may expand MDSCs in vivo (12-16). Additionally, the results from Sui et al. (11) emphasize that adjuvant-driven MDSC expansion may have a detrimental impact on vaccine-induced immune responses and highlight the need for additional investigation on the impact of vaccine adjuvants on both innate and adaptive immune responses, including regulatory responses. The impact of the route of immunization on vaccine/adjuvantinduced MDSCs will also require further evaluation. Sui et al. used a mucosal route of immunization; however, it remains to be determined whether vaccine/adjuvant-induced MDSC responses are influenced by the route of immunization.

\section{Future considerations for HIV-1 vaccine development}

The work by Sui et al. (11) has highlighted innate immune cell regulation as a potential mechanism explaining the lack of success of clinical trials that aimed to elicit $\mathrm{CD}^{+} \mathrm{T}$ cell responses that would reduce or eliminate virus replication, such as the STEP trial, which evaluated the efficacy of an adenovirus-based vaccine candidate. (17). While more studies will be required to confirm the impact of MDSCs on vaccine-elicited cellular immune responses, the data put forth by Sui et al. (11) suggest that assessing the myeloid cell expansion in response to vaccination could be critical in improving effective $\mathrm{CD}^{+} \mathrm{T}$ cell responses elicited by HIV-1 vaccination. As current HIV-1 vaccine candidates, such as the cytomegalovirus-vectored vaccines (18), that target $T$ cell responses and the elimination of early viral reservoirs move forward in preclinical and clinical vaccine trials, the impact of innate regulation on vaccine-elicited $\mathrm{T}$ cell responses should be considered in attempts to improve the effectiveness of these HIV-1 vaccines. Development of vaccines that target both cellular and humoral immune responses to block HIV-1 infection and eliminate early viral reservoirs will require researchers in the HIV-1 vaccine field to consider the complex immunologic principles that 
regulate the $T$ and $B$ cell responses that effective vaccines seek to generate.

Address correspondence to: Sallie R. Permar, Human Vaccine Institute, Duke University Medical Center, Box 103020, Durham, North Carolina 27710, USA. Phone: 919.684.2551; Fax: 919.668.4859; E-mail: sallie.permar@duke.edu.

1. Excler JL, Robb ML, Kim JH. HIV-1 vaccines: challenges and new perspectives. Hum Vaccin Immunother. doi: $10.4161 /$ hv.28462.

2. Haynes BF, McElrath MJ. Progress in HIV-1 vaccine development. Curr Opin HIV AIDS. 2013; 8(4):326-332.

3. Moody MA, et al. Toll-like receptor $7 / 8$ (TLR7/8) and TLR9 agonists cooperate to enhance HIV-1 envelope antibody responses in rhesus macaques. J Virol. 2014;88(6):3329-3339.

4. Buffa V, Klein K, Fischetti L, Shattock RJ. Evaluation of TLR agonists as potential mucosal adjuvants for HIV gp140 and tetanus toxoid in mice. PLoS One. 2012;7(12):e50529.

5. Fouda GG, et al. Mucosal immunization of lactating female rhesus monkeys with a transmitted/ founder HIV-1 envelope induces strong Envspecific IgA antibody responses in breast milk. JVirol. 2013;87(12):6986-6999.

6. Staats HF, et al. Cytokine requirements for induction of systemic and mucosal CTL after nasal immunization. J Immunol. 2001;167(9):5386-5394.

7. Bradney CP, Sempowski GD, Liao HX, Haynes BF, Staats HF. Cytokines as adjuvants for the induction of anti-human immunodeficiency virus peptide immunoglobulin $\mathrm{G}$ (IgG) and IgA antibodies in serum and mucosal secretions after nasal immunization. J Virol. 2002; 76(2):517-524

8. Egan MA, et al. A comparative evaluation of nasal and parenteral vaccine adjuvants to elicit systemic and mucosal HIV-1 peptide-specific humoral immune responses in cynomolgus macaques. Vaccine. 2004;22(27-28):3774-3788.

9. Ackerman ME, Dugast AS, Alter G. Emerging concepts on the role of innate immunity in the prevention and control of HIV infection. Annu Rev Med. 2012;63:113-130.

10. Altfeld M, Fadda L, Frleta D, Bhardwaj N. DCs and NK cells: critical effectors in the immune response to HIV-1. Nat Rev Immunol. 2011;11(3):176-186.

11. Sui Y, et al. Vaccine-induced myeloid cell population dampens protective immunity to SIV. J Clin Invest. 2014;124(6):2538-2549.

12. Ray A, Chakraborty K, Ray P. Immunosuppressive
MDSCs induced by TLR signaling during infection and role in resolution of inflammation. Front Cell Infect Microbiol. 2013;3:52.

13. Dang Y, et al. Dendritic cell-activating vaccine adjuvants differ in the ability to elicit antitumor immunity due to an adjuvant-specific induction of immunosuppressive cells. Clin Cancer Res. 2012; 18(11):3122-3131.

14. Bunt SK, Clements VK, Hanson EM, Sinha P, Ostrand-Rosenberg S. Inflammation enhances myeloid-derived suppressor cell cross-talk by signaling through Toll-like receptor 4. J Lenkoc Biol. 2009;85(6):996-1004.

15. Rieber N, et al. Flagellin induces myeloid-derived suppressor cells: implications for Pseudomonas aeruginosa infection in cystic fibrosis lung disease. J Immunol. 2013;190(3):1276-1284.

16. Garg A, Spector SA. HIV type 1 gp120-induced expansion of myeloid derived suppressor cells is dependent on interleukin 6 and suppresses immunity. J Infect Dis. 2014;209(3):441-451.

17. Buchbinder SP, et al. Efficacy assessment of a cell-mediated immunity HIV-1 vaccine (the Step Study): a double-blind, randomised, placebocontrolled, test-of-concept trial. Lancet. 2008; 372(9653):1881-1893.

18. Hansen SG, et al. Profound early control of highly pathogenic SIV by an effector memory T-cell vaccine. Nature. 2011;473(7348):523-527.

\section{Inhibiting HDAC for human hematopoietic stem cell expansion}

Hal E. Broxmeyer

Department of Microbiology and Immunology, Indiana University School of Medicine, Indianapolis, Indiana, USA.

In this issue of the JCI, Chaurasia and colleagues report an impressive ex vivo expansion of HSCs from human cord blood (CB) using cytokines and altering epigenetic modifications. The application of this protocol provides information that has potential for clinical consideration. The enhanced expansion of CB HSCs is a substantial advance over recent work from the Chaurasia and Hoffman group, in which ex vivo production of human erythroid progenitor cells from CB was promoted by chromatin modification. Moreover, this study takes advantage of information from the rapidly emerging, but not yet fully elucidated, field of epigenetics.

\section{Limited HSCs in cord blood prevent broad clinical use}

The limited number of HSCs in single cord blood (CB) collections has been problematic for efficient engraftment in adult patients (1), hence the efforts of numerous groups to compensate for low HSC numbers by attempting to transplant double CB units, expand HSCs from CB ex vivo,

Conflict of interest: Hal E. Broxmeyer is on the Medical Scientific Advisory Board of Corduse, a cord blood banking company, and in the past has consulted for Fate Therapeutics and has received income from these companies.

Citation for this article: J Clin Invest. doi:10.1172/ JCI75803. increase the homing capabilities of HSCs through priming and other maneuvers, or modulate the recipient's microenvironment for more efficient engraftment (1). A number of efforts have been put forth to expand human HSCs ex vivo (2), with limited success.

\section{HDAC inhibition dramatically improves CB HSC expansion}

Epigenetics is a high-profile area of investigation (3). In this issue, Chaurasia et al. (4) followed up on their previous studies (5) by focusing on means to more effectively expand HSC populations isolated from $\mathrm{CB}$ using histone deacetylase inhib- itors (HDACIs), primarily valproic acid (VPA), either in the context of cytokine-primed CB cells or with greater effect in addition to cytokines for the 7-day period of ex vivo cell culture (Figure 1). The cytokine cocktail included stem cell factor, FLT3 ligand, thrombopoietin, and IL-3 which, together in the presence of VPA, produced a phenomenal expansion of engraftable HSCs as assayed by stateof-the-art procedures. Chaurasia and colleagues evaluated and quantitated human HSC engraftment and repopulation in sublethally irradiated NOD/SCID IL-2 receptor $\gamma \mathrm{c}$-null (NSG) mice (4). Using limiting cell dilution analysis, Chaurasia et al. determined that the frequency of SCID-repopulating cells (SRCs) after ex vivo culture of CB cells with cytokines and VPA was 1 SRC in every 31 cells, while CB cells cultured with only cytokines produced 1 SRC in 9,225 cells, and input (unexpanded CB cells) had 1 SRC in 1,115 cells. This translated to respective numbers of 32,258 SRCs from ex vivo-cultured $\mathrm{CB}$ cells in the presence of cytokines plus 